\title{
Fusion of Multi-focus Color Images Based on Wavelet Transform and Curvelet T./ransform
}

\author{
دمج الصور الملونة متعددة التركيز باستخدام تحويلى المويجات \\ و المنحنبات
}

\author{
Hossam El-Din Moustafa, Yasmeen Abdullah \\ Faculty of Engineering, Mansoura University, Mansoura, Egypt, \\ E-mail: hossam_moustafa@hotmail.com
}

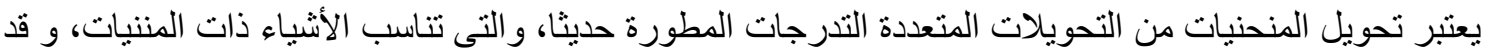

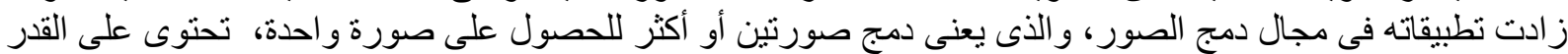

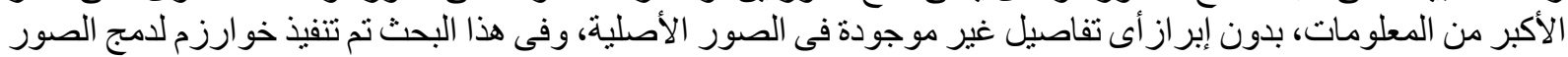

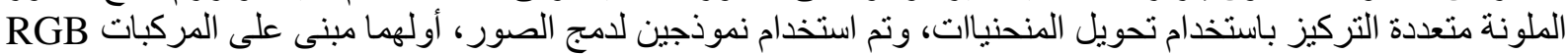

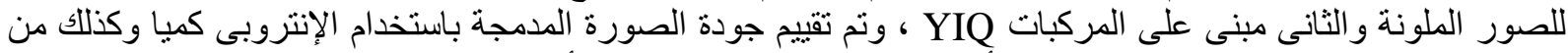

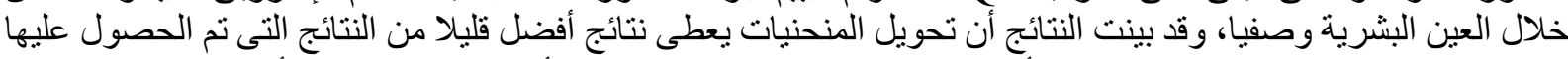

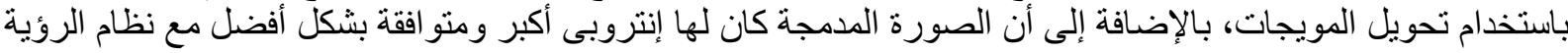

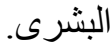

\begin{abstract}
Curvelet transform is a recently-developed multi-scale transform, which is more suitable for objects with curves. Applications of the Curvelet transform have increased rapidly in the field of image fusion. Image fusion means the combination of two images into a single image that has the maximum information content without producing details that are non-existent in the given images. In the present work an algorithm for multi-focus color image fusion based on the Curvelet transform is implemented, analyzed, and compared with a Waveletbased fusion algorithm. Two models for color image fusion are presented. The first is based on the RGB components of the color image, while the other is based on the YIQ color model. The quality of the fused color image is evaluated by entropy and a human perception inspired quality metric. Experimental results have shown that the Curvelet based image fusion algorithm provides a slightly better fused image than the Wavelet algorithm. In addition, the fused image has a higher value of entropy and is more accord with the human visual system.
\end{abstract}

\section{Keywords}

Image fusion - Multi-focus Images - Wavelet Transform - Curvelet Transform

\section{Introduction}

Image fusion is the process of generating a single image that contains a more accurate description of the scene than multiple images from different sources [1]. Image fusion aims to generate a single image which contains more precise reliable visualization of the objects than any of the source images. Such a fused image should provide extended information and better perception for human vision or computerized vision tasks. The way the sources combine their information depends upon the nature of the images and the way they have been acquired. Images can be fused in the spatial domain or in other transformed domains. Image fusion has been used in application areas such as remote sensing and, intelligent robots, military applications, and medical imaging [2].

Assessment of quality of the fused images is an important issue. However, this task is difficult as the reference image is usually unavailable [3]. In addition, previous researches have shown that a 
single measurement cannot effectively evaluate the performance of the fusion technique. So, in many cases, the human visual sense (HVS) can be used as an effective performance criterion. In the present work, the entropy will be used as an objective measure for the quality of the fused image.

The next section provides an overview about the Wavelet fusion algorithm. In section III, a brief background about the Curvelet transform is introduced. Section IV presents the concept of color-based image fusion models. In section $\mathrm{V}$, the proposed fusion algorithms are applied to a set of multifocus color images and compared using both entropy and human visual sense.

\section{Wavelet transform for image fusion}

The most common form of transform type image fusion algorithms is the Wavelet fusion algorithm due to its simplicity and its ability to preserve time and frequency details of the images to be fused [4]. In common with all transformdomain fusion techniques, the transformed images are combined in the Wavelet domain using a pre-defined fusion rule, then transformed back to the spatial domain to give the resulting fused image [5].

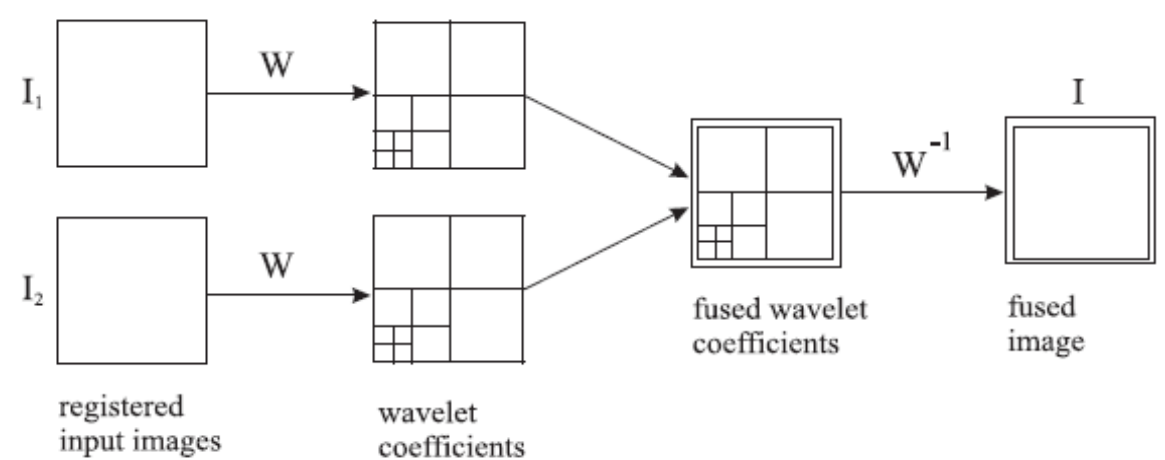

Fig.1: Wavelet transform-based image fusion

Wavelet transform fusion is more formally defined by considering the Wavelet transforms $\mathrm{w}$ of the two registered input images $\mathrm{I} 1(\mathrm{x}, \mathrm{y})$ and $\mathrm{I} 2(\mathrm{x}, \mathrm{y})$ together with the fusion rule $\varphi$. Then, the inverse Wavelet transform w-1 is computed, and the fused image $\mathrm{I}(\mathrm{x}, \mathrm{y})$ is reconstructed as shown in the following equation. This process is depicted in Fig. 1.

$$
I(x, y)=w^{-1}\left(\varphi\left(w\left(I_{1}(x, y)\right), w\left(I_{2}(x, y)\right)\right)\right)
$$

\section{The curvelet transform}

Candes and Donoho developed a multi-scale transform which they called the Curvelet transform. Motivated by the needs of image analysis, it was nevertheless first proposed in the context of objects $\mathrm{f}(\mathrm{x} 1, \mathrm{x} 2)$ defined on the continuum plane $\left(x_{1}, x_{2}\right) \in R^{2}$. The transform was designed to represent edges and other singularities along curves much more efficiently than traditional transforms, i.e. using many fewer coefficients for a given accuracy of 
reconstruction as shown in Fig. 2. Roughly speaking, to represent an edge to squared error $1 / N$ requires $1 / N$ Wavelets and only about $1 / \sqrt{N}$ Curvelets [6]. According to approximation theory, the Curvelet transform is more efficient and faster than traditional transforms like Fourier and Wavelet transforms.

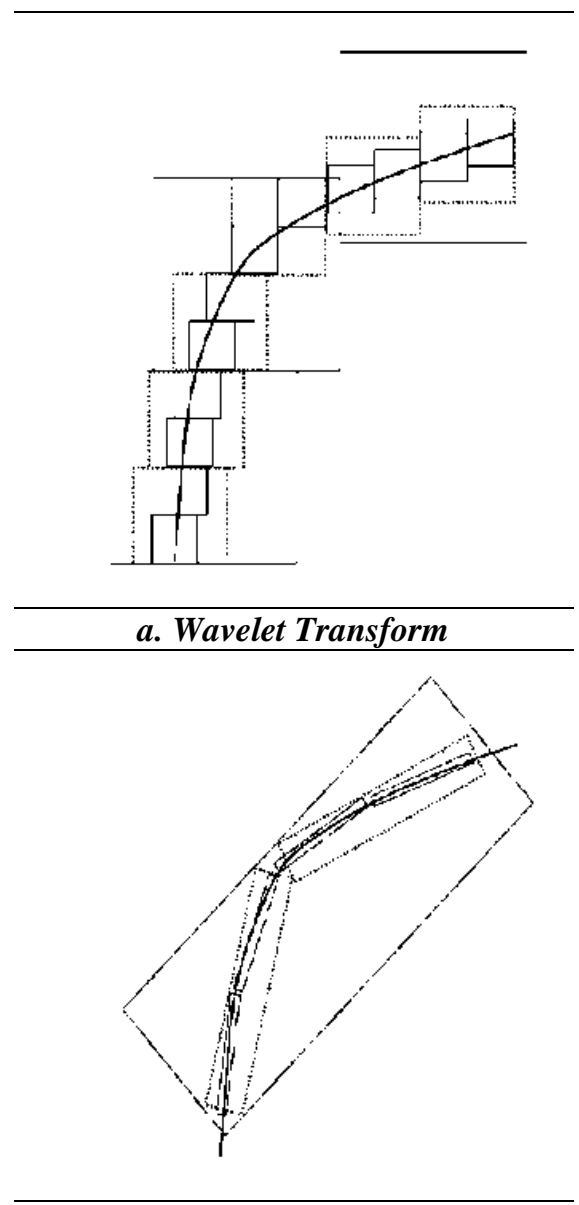

b. Curvelet Transform

Fig. 2 Wavelet coefficients versus Curvelet coefficients

The Curvelet transform is suited for objects which are smooth away from discontinuities across curves. Using Wavelets, it affects only a limited number of coefficients. Discontinuities across a simple curve affect all the Wavelet coefficients on the curve. Hence the Wavelet transform doesn't handle curves discontinuities well. Curvelets are designed to handle curves using only a small number of coefficients as mentioned before. Hence the Curvelet transform handles curve discontinuities well. Curvelet transform has applications in many fields such as image processing including image denoising, image fusion, seismic exploration, and turbulence analysis in fluid mechanics [7].The Curvelet transform includes four stages [6]:

1. Sub-band decomposition

2. Smooth partitioning

3. Renormalization

4. Ridgelet analysis

The procedure of obtaining inverse Curvelet transform can be summarized by simply reversing the previously explained steps.

The specific operational procedure for the Curvelet-based image fusion approach is now given. The algorithm is summarized as follows:

1. The two input images are first registered.

2. The Curvelet transform steps are performed for both images (each input image is analysed and a set of Curvelet coefficients are generated).

3. The maximum frequency fusion rule or any other rule for image fusion is used for the fusion of the Curvelet coefficients.

4. The inverse Curvelet transform step is performed (The fused coefficients are subjected to the inverse Curvelet transform) to obtain the fused image.

These steps are expected to merge the details in both images into a single image with much more details. 


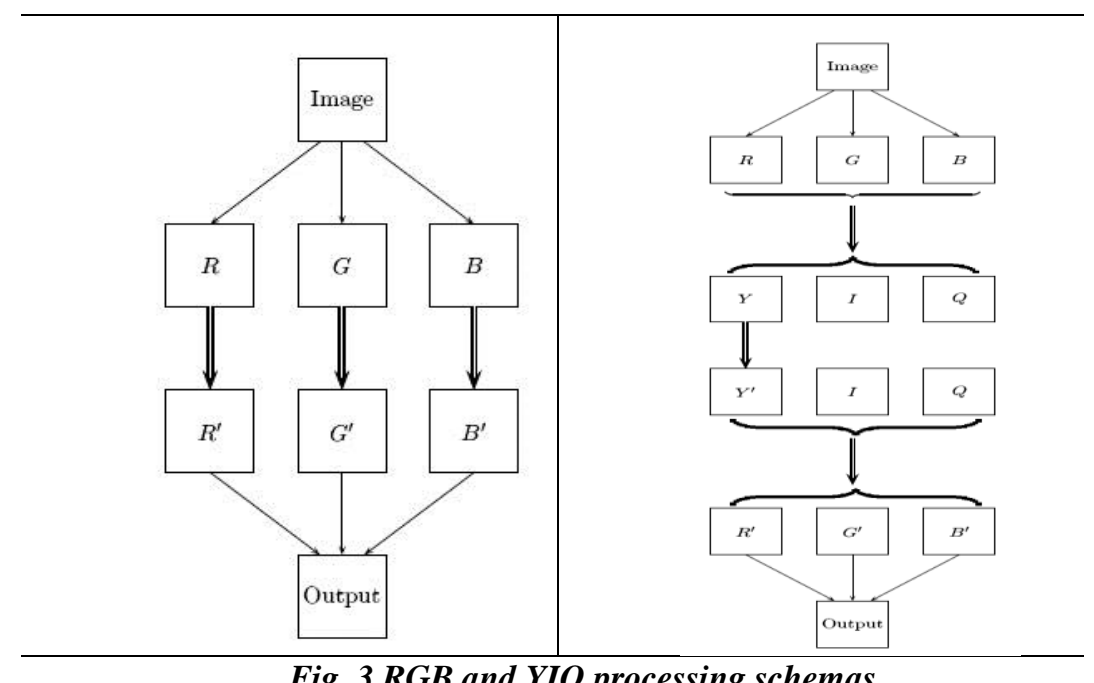

\section{Color-Based Image Fusion}

There are two methods that can be used for processing of color images [8]; process each R, G, B matrix separately or transform the color space to one in which the intensity is separated from the color, and process the intensity component only. Schemas for these methods are shown in Fig. 3.

\section{A.Fusion in RGB Model}

The basic idea is to get the matrices $\mathrm{R}, \mathrm{G}, \mathrm{B}$ of the two images to be fused i.e. convert each 3-dimensional input image to three 2-dimensional images. Then, the $\mathrm{R}$ matrix of the first image is fused with the $R$ matrix of the second image, $G$ with $G$, and $B$ with $B$, using the two fusion algorithms. Finally put the resultant fused $\mathrm{R}, \mathrm{G}$, and $\mathrm{B}$ matrices back into a single 3dimensional color image.

\section{B. Fusion in YIQ Model}

The fusion process of two images in YIQ model starts with converting the two input images from RGB model to YIQ model. Then the intensity matrix $\mathrm{Y}$ is separated from the color matrices I and Q for each image. The fusion process is carried out on the intensity component $(\mathrm{Y})$ of the two images using the two fusion algorithms. The fused $\mathrm{Y}$ matrix resulting from fusion using each algorithm and the average of matrices $\mathrm{I}$ and $\mathrm{Q}$ of the two input images are gathered back into a single 3-dimensional image. Finally, the resultant images are converted to RGB model.

\section{C.Performance Measures}

The widespread use of image fusion methods has led to an increasing need for pertinent performance or quality assessment tools in order to compare results obtained with different algorithms or to obtain an optimal setting of parameters for a given fusion algorithm. In the present work, the entropy was used to evaluate the performance of the Curvelet fusion algorithm and to compare it with Wavelet fusion algorithm. The Entropy, H, of an image is a measure of information content [9]. It is the average number of bits needed to quantize the intensities in the image. It is defined as:

$$
H=-\sum_{g=0}^{L-1} p(g) \log _{2} p(g)
$$

\section{Fusion of Multi-focus Images}

There are sensors which cannot generate images of all objects at various distances with equal clarity (e.g. camera 
with finite depth of field, light optical microscope, etc.). Thus several images of a scene are captured, with focus on different parts of it. The acquired images are complementary in many ways and a single one of them is not sufficient in terms of their respective information content [9]. However, viewing a series of such images separately and individually is not very useful and convenient. The advantages of multi-focus data can be fully exploited by integrating the sharply focused regions seen in the different images. This process is known as multi-focus image fusion. Both the reliability of redundant information and the quality of complementary information present in the constituent images are improved in the fused image. So, it gives a better view for human and/or machine perception.

\section{A.Fusion of Color Images}

Both fusion in RGB model and fusion in YIQ model are applied for fusing multi-focus color images. The two fusion algorithms, previously explained, are used in the fusion process. The two methods are compared using the entropy performance measure. The original multi-focus color images are shown in Fig. 4.

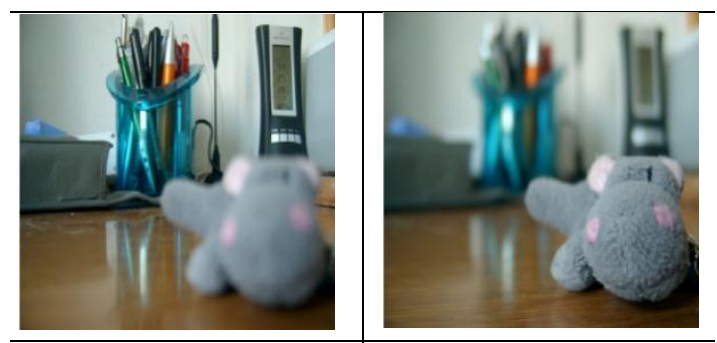

First image $\quad$ Second image

Fig. 4 Multi-focus color images

\section{B. Fusion in RGB Model}

Figures 5(a), 5(b), 5(c) show the R, $\mathrm{G}$, and $\mathrm{B}$ matrices of the two multi-focus input color images respectively as well as their fused image using two different image fusion techniques. The fused R, G, $\mathrm{B}$ matrices are rearranged back into a single 3 dimensional image. Figure 5(d) shows the fused multi-focus color images using RGB model.

\section{Fusion in YIQ Model}

Figure 6 shows the $\mathrm{Y}, \mathrm{I}$, and $\mathrm{Q}$ matrices of the two multi-focus input color image. Figure 7(a) shows the fused $\mathrm{Y}$ matrices using two different image fusion techniques. The fused $\mathrm{Y}$ matrix resulting from fusion using each algorithm and the average of matrices $\mathrm{I}$ and $\mathrm{Q}$ of the two input images are gathered back into a single 3 dimensional image. The resultant images are converted to RGB model. Figure 7(b) shows the fused multi-focus color images using YIQ model.

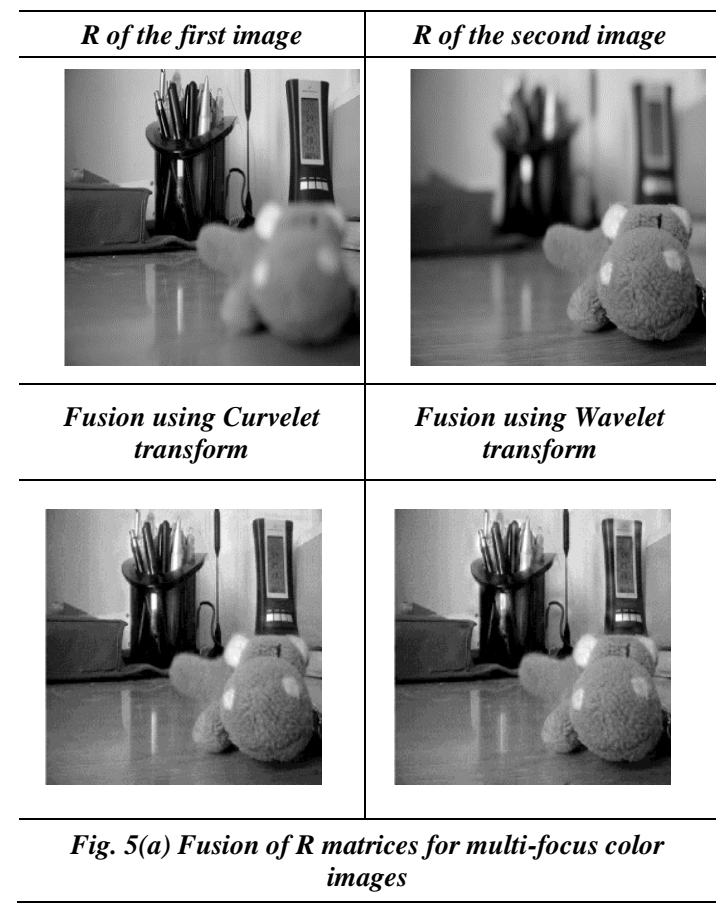




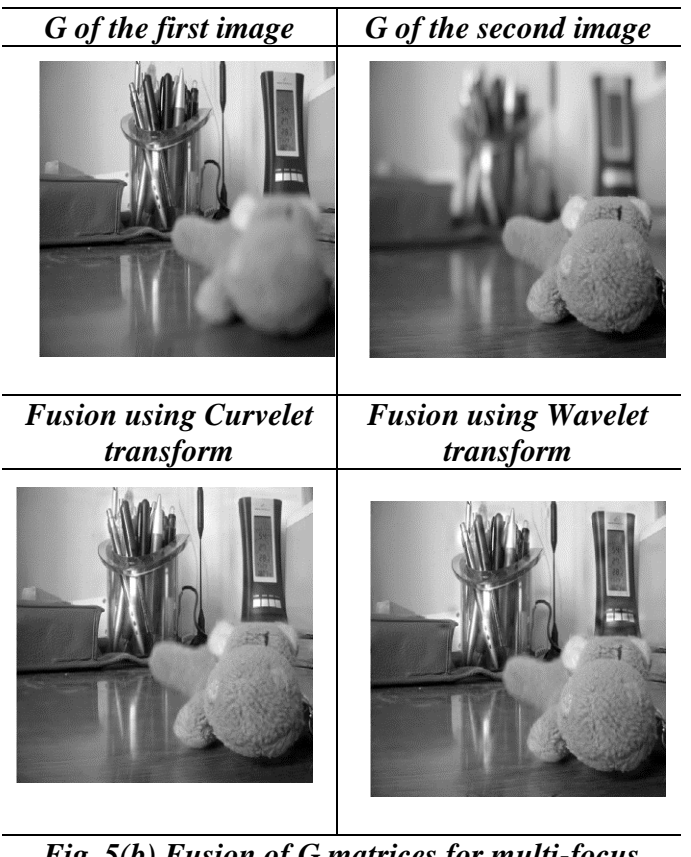

Fig. 5(b) Fusion of G matrices for multi-focus color images

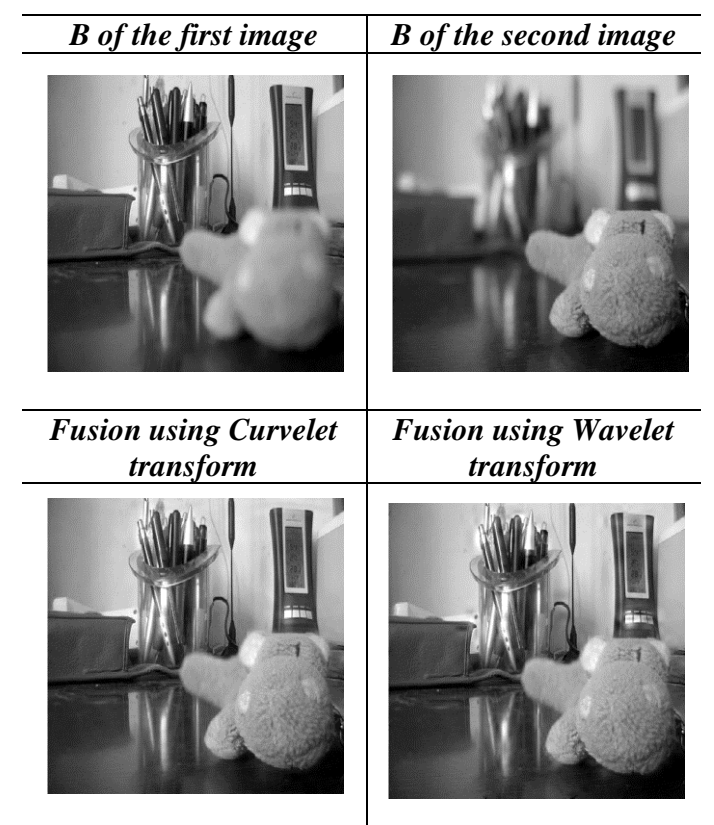

Fig. 5(c) Fusion of B matrices for multi-focus color images

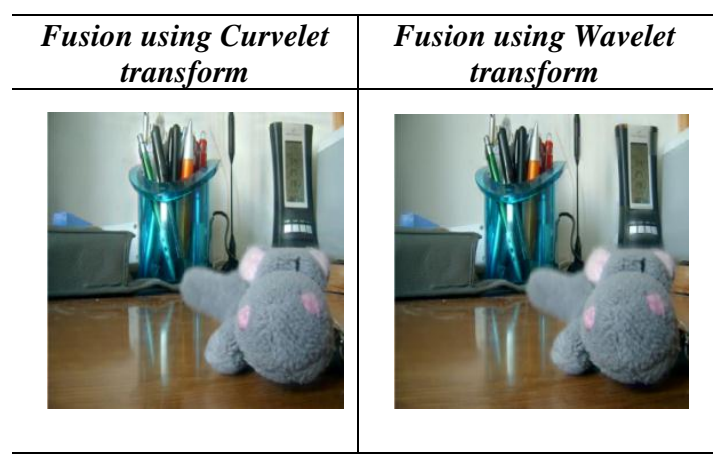

Fig. 5(d) Fused multi-focus color images using RGB model

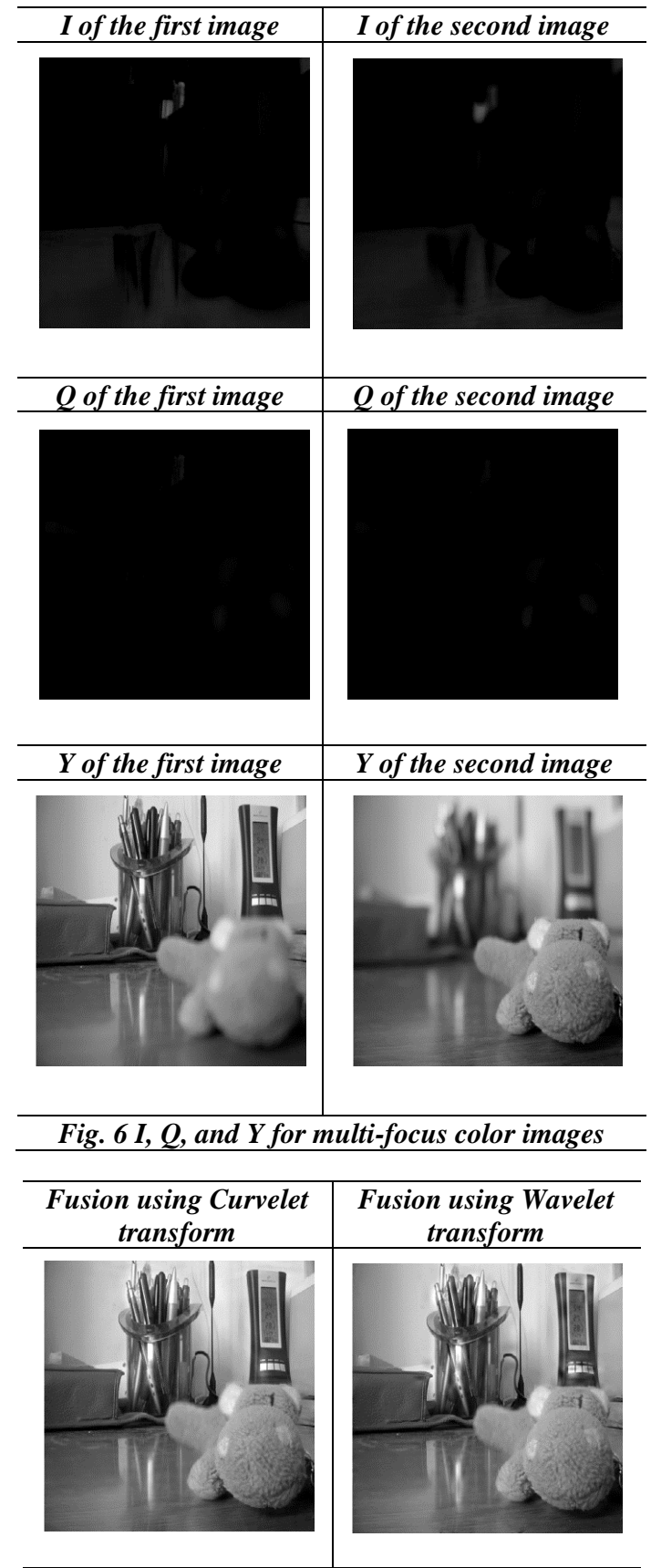

Fig. 7(a) Fusion of Y matrices of the multi-focus color images

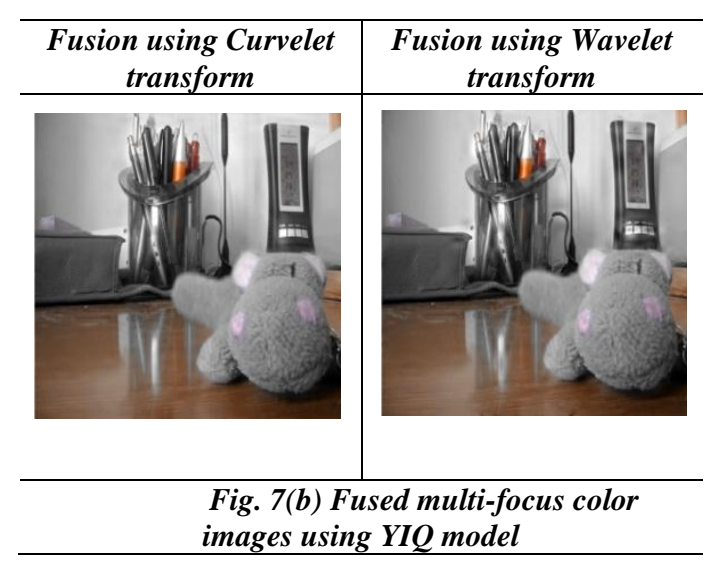


The entropy performance measure and the processing time using personal computer with Core 2 Duo $2.4 \mathrm{GHz}$ processor are used to compare between fusion in RGB model and fusion in YIQ model for each fusion algorithm. Table 1 gives a comparison between fusion in RGB model and fusion in YIQ model for multifocus images and gives a comparison between all fusion techniques.

Table 1: Entropy and processing time for fused images of multi-focus color images

\begin{tabular}{|c|c|c|c|c|}
\hline Algorithm & \multicolumn{2}{|c|}{ Fusion in RGB } & \multicolumn{2}{c|}{ Fusion in YIQ } \\
\hline & Entropy & $\begin{array}{c}\text { Processing time } \\
\text { in seconds }\end{array}$ & Entropy & $\begin{array}{c}\text { Processing time in } \\
\text { seconds }\end{array}$ \\
\hline Wavelet & 7.8161 & 4.3422 & 7.7719 & 1.4983 \\
\hline Curvelet & 7.8403 & 4.3023 & 7.7921 & 1.4882 \\
\hline
\end{tabular}

The values of the first input image entropy and second input image entropy are 7.8129 and 7.7373 respectively (average entropy $=7.7751$ ).

According to visual perception, the fused image has been significantly improved using the two methods for both fusion techniques. It is noticed that the fusion in RGB model has better eye perception than fusion in YIQ model. The Entropy performance measure has shown that fusion in RGB model provides a slightly better fused image than fusion in YIQ model. Fusion in YIQ model has the advantage of less processing time due to the reduced number of fusion processes. Results have shown that the Curvelet technique has higher performance than Wavelet technique, thus it can be adopted for applications including multi-focus color images.

\section{Conclusion}

Two image fusion algorithms have been proposed and compared. They are based on the Wavelet transform and the Curvelet transform. These algorithms were applied on a set multi-focus color images using two color models; RGB and YIQ. The entropy was used to assess the effectiveness of the two image fusion algorithms for both color models. Results have proved that there is a slight difference between the performances of the two fusion algorithms. It has been shown also that the best performance criterion should be linked with the specific application.
According to visual perception, the fused image has been significantly improved using both fusion techniques. Image fusion with Curvelet transform has given curved visual details better than those given by the Wavelet fusion algorithm.

\section{References}

[1] G. Liu, Z. Jing, S. Sun "Image fusion based on an expectation maximization algorithm" Optical Engineering, Vol. 44, No. 7, 2005.

[2] M. I. Smith, J. P. Heather, "Review of Image Fusion Technology in 2005," Proceedings of the SPIE, Volume 5782, pp. 29-45, 2005.

[3] A. Malviya, S.G. Bhirud, "Objective Criterion for Performance Evaluation of Image Fusion Techniques" International Journal of Computer Applications, Vol.1, No. 25, 2010.

[4] F. E. Ali, I. M. El-Dokany, A. A. Saad and F. E. Abd El-Samie "Curvelet Fusion of MR and CT images", Progress In Electromagnetics Research, Vol. 3, pp. 215-224, 2008.

[5] P. Hill, N. Canagarajah D. Bull "Image Fusion Using Complex Wavelets", Technical report, Dept. of Electrical and Electronic Engineering, The University of Bristol, UK, 2002.

[6] D. L. Donoho \& M. R. Duncan "Digital Curvelet Transform: Strategy, Implementation and Experiments", Technical report, Department of Statistics, Stanford University November, 1999. 
[7] L. Demanet, D. Donoho, L. Ying, "Fast Discrete Curvelet Transforms", Multiscale Model Simul., Vol. 5, No. 3, pp. 861-899, 2006.

[8] A. McAndrew, "An Introduction to Digital Image Processing with Matlab", School of Computer Science and Mathematics Victoria University of Technology, 2004.
[9] I. De, B. Chanda, "A Simple and Efficient Algorithm for Multi-focus Image Fusion Using Morphological Wavelets", Signal Processing, Vol. 86, pp. 924-936, 2006. 